\title{
Exploration Disaster Source of Mine Water by Electromagnetic Radiation
}

\author{
Benyu $\mathrm{Su}^{1,2}$, Zhixiong $\mathrm{Li}^{3,4}$, Rongyao $\mathrm{Li}^{1}$, Rongfu Rao ${ }^{1}$, Jingcun $\mathrm{Yu}^{1, *}$ \\ ${ }^{l}$ Institute of Mine Water Hazards Prevention and Controlling Technology, School of Resources and \\ Geosciences, China University of Mining and Technology, \\ Xuzhou, Jiangsu Province 221116, China \\ ${ }^{2}$ Shaanxi Provincial Key Laboratory of Geological Support for Coal Green Exploitation, \\ Xi'an 710054, China \\ ${ }^{3}$ School of Engineering, Ocean University of China, \\ Tsingtao 266100, China \\ ${ }^{4}$ Yonsei Frontier Lab, Yonsei University, \\ 50 Yonsei-ro, Seodaemun-gu, Seoul 03722, Republic of Korea \\ yjccumt@gmail.com
}

\begin{abstract}
Rock burst is a common typical geological hazard in deep underground mining. Before the rock mass explosion, electromagnetic energy will radiate outward during the deformation and rupture of the coal rocks. Hence, it is possible to use the electromagnetic radiation to predict geological disasters in coal mines. A challenging task using the active source electromagnetic survey technique is to detect geological anomalies, such as disaster water sources and geological structures. To this end, this paper proposes a new electromagnetic radiation solution based on the forward and inversion theory to detect geological anomalies in the coal seam. Based on typical coal mine geological models, the forward modelling and inversion modelling have been performed, respectively. The forward modelling explained the geological anomalies inside the coal seam, which were very sensitive to the response of the radiated electromagnetic field; especially, for the water-bearing geological anomalies. The inversion modelling discovered that the inversion geo-electric parameter distribution agreed well with the actual model. As a result, the proposed method is feasible for geological anomalies detection.
\end{abstract}

Keywords-Coal mining safety; Rock burst; Electromagnetic radiation; Electromagnetic scattering.

\section{INTRODUCTION}

Electromagnetic waves are used to detect the obscured and remote regions based on the imaging techniques [1]-[7]. For example, oil and gas exploration in oil industry [8], nondestructive evaluation in industrial structures [9], and physical examinations [10]. In fact, the electromagnetic theory is widely used in the field of coal mine safety [11]. China is one of the countries with the most severe rock burst, where serious restrict has been made to ensure safety and

Manuscript received 25 March, 2020; accepted 14 August, 2020.

This research was funded by National Natural Science Foundation under Grant No. 41974151; Jiangsu province Natural Science Foundation under Grant No. BK20181360; National Key Research And Development Program of China under Grant No. 2018YFC0807804-02; Open Fund of Shaanxi Key Laboratory of Geological Support for Coal Green Exploitation under Grant No. DZBZ2020-06; Shaanxi Province Technology Innovation Guidance under Grant No. 2020CGHJ-005, and the Priority Academic Program Development of Jiangsu Higher Education Institutions. efficiency of coal mine production. As an effective geophysical monitoring method, the electromagnetic radiation technology has been applied to most mines with serious rock burst in China [12], e.g., rock burst prediction [13]. However, the electromagnetic radiation can be used only for qualitative analysis. For example, the intensity of electromagnetic radiation reflects the stress and burst degree of the coal rock mass and the amount of pulses mainly reflects the rock cracks [14]. Therefore, the burst degree of the coal rock mass can be predicted using the characteristics of the electromagnetic radiation field. The problem is that it cannot accurately locate the area of rock fracture. For another example, the electromagnetic geophysical methods are employed to detect geological anomalous structures and disaster sources of water [15]-[17]. However, existing methods are only applicable to low frequency range and cannot accurately locate the position of the anomalous bodies.

In fact, the rock burst often occurs in a sudden or violent way in underground rock masses. Although the failure process of rocks is usually characterized by crack initiation, propagation, and coalescence with associated damage and evolution, the developing time of the whole process is very short. Therefore, the radiation electromagnetic wave exhibits high frequency characteristics. According to the Maxwell theory, the responses of high frequency electromagnetic waves are mainly associated with medium permittivity [18], which is very sensitive to bearing of coal rock [19]. Coincidentally, the medium permittivity is very sensitive for the rock watery. As a result, it is reasonable to analyse rock permittivity to detect geological anomalies.

This paper aims to study the distribution of the geoelectrical parameters using electromagnetic radiation by electromagnetic inversion method. In the research process, the permittivity of the electromagnetic waves is analysed. Numerical analysis results demonstrate that the electromagnetic inverse scattering is effective to locate disaster water source. 


\section{SCATTERING FORWARD PROBLEM}

The total electromagnetic fields satisfy the Maxwell equations in the presence of the scatter:

$$
\begin{gathered}
\nabla \times H^{t}=-i \omega \varepsilon E^{t}, \\
\nabla \times E^{t}=i \omega \mu H^{t} .
\end{gathered}
$$

In fact, the total field includes two parts: incident field and scattered field

$$
\begin{aligned}
& E^{t}=E^{i}+E,{ }^{s} \\
& H^{t}=H^{i}+H^{s} .
\end{aligned}
$$

In addition, the incident field also satisfies the Maxwell equations:

$$
\begin{gathered}
\nabla \times H^{\mathrm{i}}=-i \omega \varepsilon E^{\mathrm{i}}, \\
\nabla \times E^{\mathrm{i}}=i \omega \mu H^{\mathrm{i}} .
\end{gathered}
$$

Combining (3)-(6), the following formulas can be obtained:

$$
\begin{gathered}
\nabla \times H^{\mathrm{s}}=-i \omega \varepsilon E^{\mathrm{s}}-i \omega\left(\varepsilon_{a}-\varepsilon\right) E^{t}, \\
\nabla \times E^{s}=i \omega \mu H^{s}-\left\lfloor-i \omega\left(\mu_{a}-\mu\right) H^{t}\right\rfloor .
\end{gathered}
$$

For (7) and (8), the second item in the right part can be written as:

$$
\begin{gathered}
J=-i \omega\left(\varepsilon_{a}-\varepsilon\right) E, \\
K=-i \omega\left(\mu_{a}-\mu\right) H .
\end{gathered}
$$

Equation (9) plays the role of electric current density and (10) plays the role of magnetic current density in the Maxwell equations.

However, coal rock is usually non-magnetic, which means that $\mu_{a}=\mu=0$; therefore, (10) is equal to zero. Thus, the electromagnetic field scattering can be rewritten as:

$$
\begin{gathered}
\nabla \times H^{\mathrm{s}}=-i \omega \varepsilon E^{\mathrm{s}}+J, \\
\nabla \times E^{s}=i \omega \mu H^{s} .
\end{gathered}
$$

Combining (11) and (12), it yields

$$
\nabla \times \nabla \times E^{s}-\mathrm{k} E^{s}=i \omega \mu_{0} J(r) .
$$

For an arbitrary electric current distribution $J(r)$, it is convenient to calculate its radiation fields by using the concept of the dyadic Green's function $G\left(r, r^{\prime}\right)$, which is defined to satisfy (14)

$$
\nabla \times \nabla \times \overline{\bar{G}}\left(r, r^{\prime}\right)-k^{2} \overline{\bar{G}}\left(r, r^{\prime}\right)=\overline{\bar{I}} \delta\left(r-r^{\prime}\right),
$$

where, $\overline{\bar{I}}$ is a vector. The radiation fields by the current distribution $J(r)$ can be formulated as the convolution of the dyadic Green's function with $J(r)$

$$
E(r)=i \omega \mu_{0} \iiint \overline{\bar{G}}\left(r, r^{\prime}\right) \times J\left(r^{\prime}\right) d r^{\prime} .
$$

Here, Green's function can be expressed by (16)

$$
\overline{\bar{G}}\left(r, r^{\prime}\right)=\left\lfloor\overline{\bar{I}}+\frac{1}{k^{2}} \nabla \nabla\right\rfloor g\left(r, r^{\prime}\right) .
$$

Green's function of impulse response meets the following condition

$$
\left(\nabla^{2}+k_{0}^{2}\right) g\left(r, r^{\prime}\right)=-\delta\left(r-r^{\prime}\right)
$$

Here, $g\left(r, r^{\prime}\right)=\frac{e^{i k_{0}\left|\mathbf{r}-\mathbf{r}^{\prime}\right|}}{4 \pi\left|r-r^{\prime}\right|}$.

Scattering electromagnetic field in the far zone can be calculated by

$$
\left.E^{s}(r)=k^{2}\right\rfloor g\left(r, r^{\prime}\right) J\left(r^{\prime}\right) d r^{\prime}
$$

However, the total electromagnetic field for the area scatter located in the domain is composited by two parts; one is the incident field and the other part is the scattering field as shown in (19) [20]

$$
E_{t}(r)=E_{i}(r)+E_{t}(r)=E_{i}(r)+k^{2} \int g\left(r, r^{\prime}\right) J\left(r^{\prime}\right) d r^{\prime},
$$

where $k$ is the wave number.

\section{FORWARD NUMERICAL MODELLING}

As mentioned above, mining water disaster and rock burst disaster are very common in China. Here, the distribution of the electromagnetic field caused by the rock burst and the scattering field corresponding to the geological anomalous body are studied by numerical simulations. Figure 1 shows the two-dimensional coal seam model with the size of $150 \times 200(\mathrm{~m})$. Assume that the fractured zone caused by the rock burst can radiate electromagnetic waves. In order to do numerical simulation, it is supposed that the center of the fractured zone is the coordinate origin and 16 electric dipoles are evenly fixed to detect the radiated source as shown in Fig. 1. Besides, the relative permittivity of the coal rock is set to 10 . The distribution of the radiated electromagnetic field is shown in Fig. 2, where the electromagnetic energy radiates evenly around the source. When there is a geological anomalous body in the coal seam, it will become scattered to generate a scattering field. As shown in Fig. 3, there is only one tunnel in the coal seam model and the relative permittivity of the tunnel is defined as 1. Note that the relative permittivity of background coal rock is 10. In this case, the scatter in Fig. 4 exhibits an obvious distribution of scattering field near the tunnel. Therefore, the scattering response of the coal mining tunnel is very sensitive to the radiation field caused by rock burst.

Besides the geological anomalous body of the tunnel, the geological disaster anomalous body will be much more significant for the coal mine safety, such as for coal mine water disaster source and coal mine goaf. As shown in Fig. 5 and Fig. 7, there are two water-bearing rocks with area of $50 \mathrm{~m}$ by $50 \mathrm{~m}$ in the right and in the front of the tunnel, respectively. 


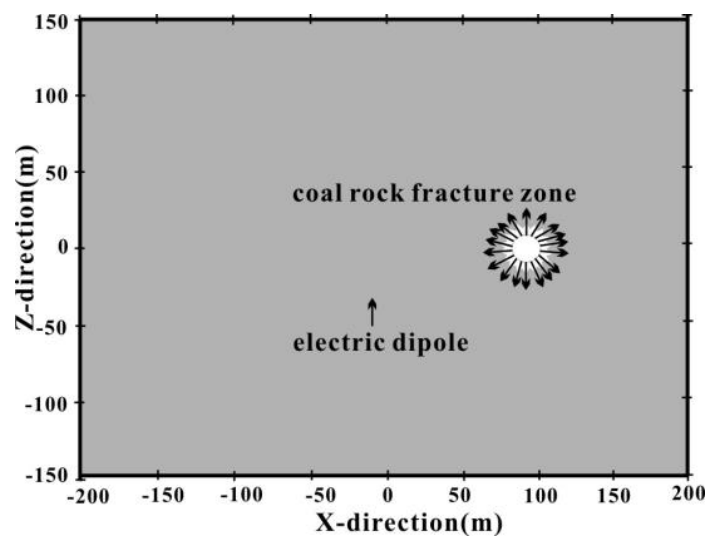

Fig. 1. Model of rock burst in the coal seam with 16 electric dipoles. The relative permittivity of coal rock is 10 .

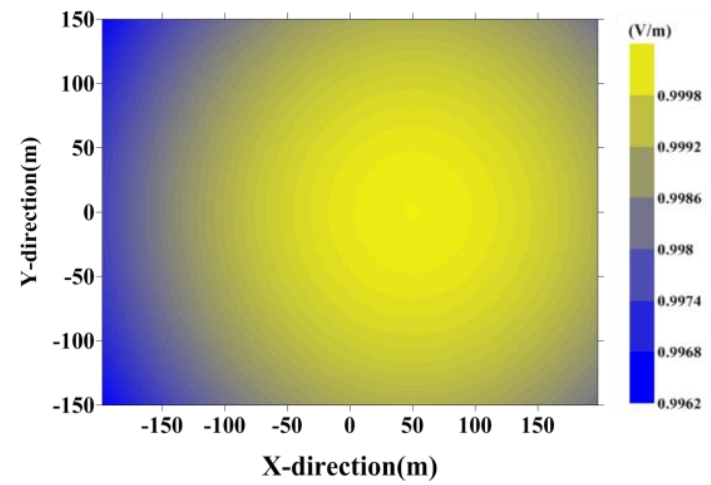

Fig. 2. Distribution of radiated electromagnetic field of Fig. 1, where the electromagnetic energy radiates evenly around the source.

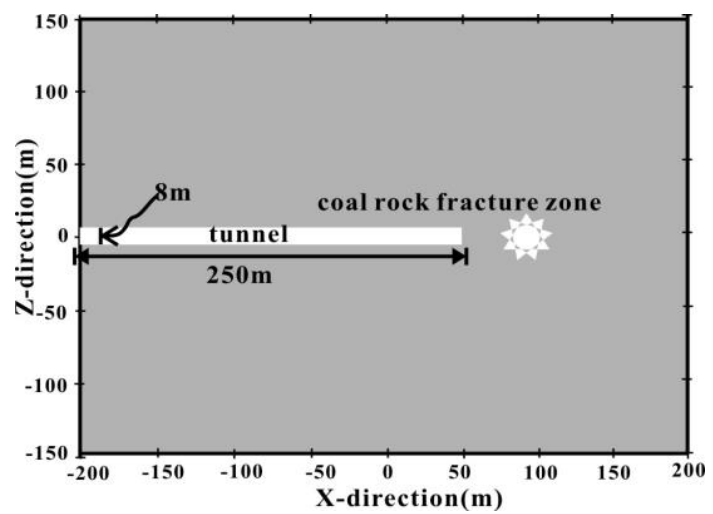

Fig. 3. Model of mine geology with tunnel and fracture zone in the front of tunnel.

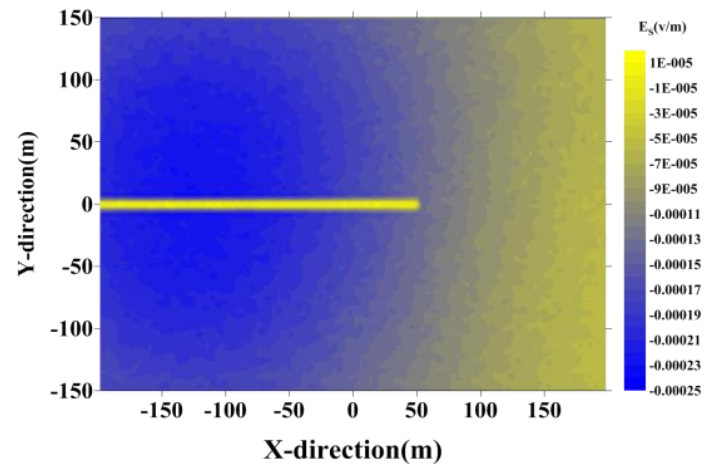

Fig. 4. Distribution of scattering electromagnetic field of Fig. 3.

The relative permittivity of the water-bearing rock, tunnel, and coal rock are 60, 1, and 10, respectively. Their responses of scattering fields are shown in Fig. 6 and Fig. 8, where both of the two cases indicate high sensitivity for the water-bearing rock. Compared with Fig. 4, because the difference of relative permittivity between coal seam and water-bearing rock is larger than that between the coal seam and tunnel, the scattering field response of the water-bearing rock is much more obvious than that of the tunnel.

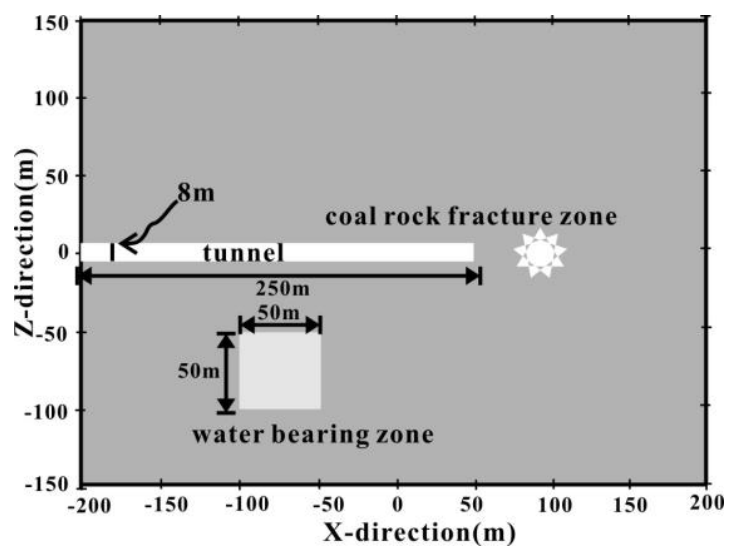

Fig. 5. Model of mine geology with tunnel and water-bearing zone in the right of the coal.

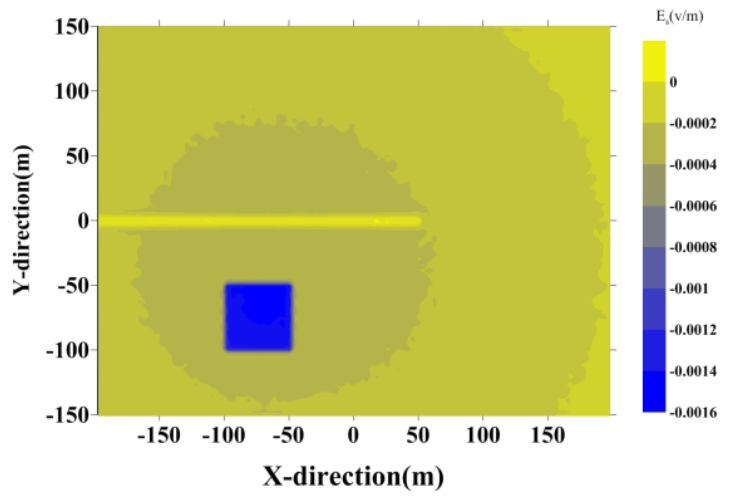

Fig. 6. Distribution of scattering electromagnetic field of Fig. 5.

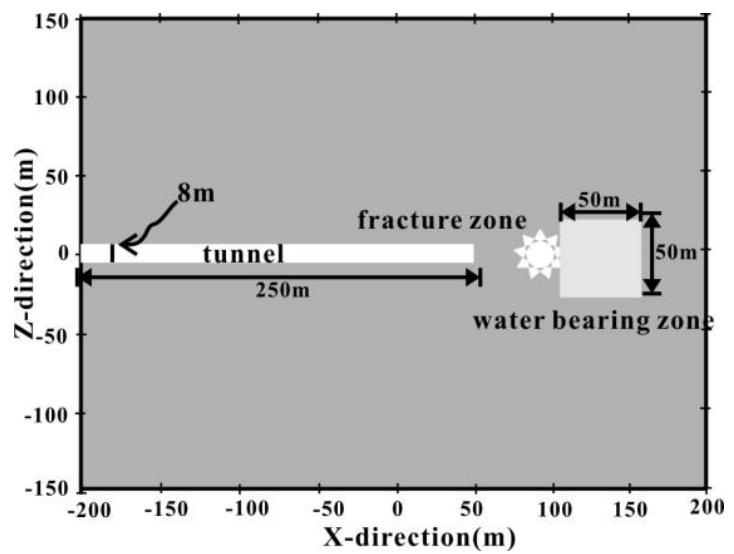

Fig. 7. Model of mine geology with tunnel and water-bearing zone in the front of the coal.

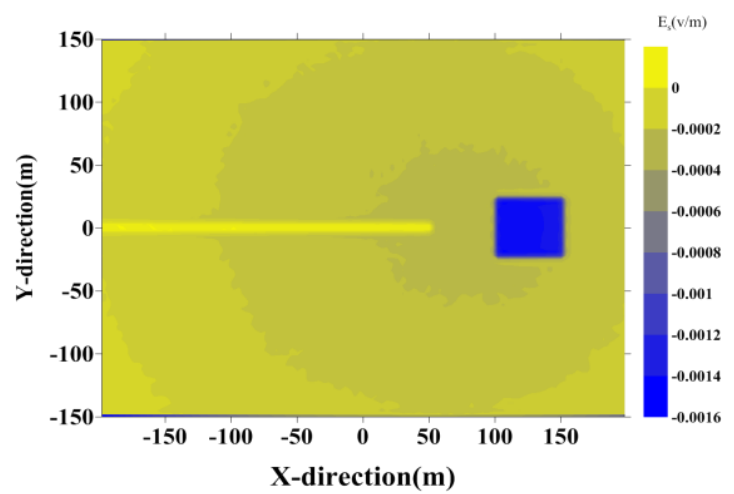

Fig. 8. Distribution of scattering electromagnetic field of Fig. 7. 


\section{SCATTERING INVERSION FORWARD PROBLEM}

For convenience, $\chi$ is introduced and it can be described using $\varepsilon_{a}$ and $\varepsilon$ as shown in (20)

$$
\chi=\left(\varepsilon_{a}-\varepsilon\right)
$$

where $\varepsilon_{a}$ denotes relative permittivity of anomalous geological body and $\varepsilon$ denotes relative permittivity of background field.

The parameter $\gamma$ is chosen to minimize the function defined as the quadratic error in the scattered field. For estimating the distribution of geo-electrical parameters, the following function has been constructed as shown in (21) [21]

$$
F(\gamma)=\left\|E_{O}^{S}-E^{s}(r)\right\|
$$

where $E^{s}(r)=G_{s}\left(\gamma G_{s}^{\dagger}\left(E^{s}\right)\right)$ and $\dagger$ indicates one electric dipole of 16 electric dipoles.

The minimum of $F(\gamma)$ requires the derivative with respect to $\gamma$ to be zero, which leads to an analytical solution of $\gamma$

$$
\gamma=\frac{<E_{o}^{s}, E^{s}(r)>}{\left\|E^{s}(r)\right\|} .
$$

Once $\gamma$ is obtained, the electric dipole generates the electrical field which can be described as

$$
E^{s}(r)=G_{s}\left(\chi E^{t}\right) \quad \in s
$$

The total field generated by all 16 electric dipoles can be calculated by

$$
J=\chi(r) E^{t}(r)
$$

The enforcement of (24) to all incidents leads to a leastsquares problem, and the solution of $\chi(r)$ can be obtained analytically

$$
\chi(r)=\frac{\sum_{p=1}^{N_{i}} J_{p}(r) \times\left[E_{p}^{t}(r)\right]^{*}}{\sum_{p=1}^{N_{i}}\left[E_{p}^{t}(r)\right]^{2}},
$$

where $\chi=\left(\varepsilon_{a}-\varepsilon\right)$. Hence, we get the new $\varepsilon_{a}$ which can be evaluated by (25) [21]

$$
g=\frac{\left\|\varepsilon_{\text {old }}-\varepsilon_{\text {new }}\right\|}{\left\|\varepsilon_{\text {old }}\right\|} .
$$

If $g$ meets the error requirement, then the computation will exit.

\section{INVERSION NUMERICAL MODELLING}

According to the actual situation encountered in the mining of coal, inversion numerical simulation mainly focuses on water-containing geological anomalous body and anhydrous goaf geological anomalous body. According to the geo-electrical parameters of a coal seam, the relative permittivity of the coal seam, water-containing coal rock, and goaf without water are respectively defined as 10,60 , and 1 .

Figure 9 shows the coal seam geological model with the area of $200 \times 200(\mathrm{~m})$ and its center coincides with defined coordinate origin. It is assumed that there is a circular watercontaining anomalous body with $10 \mathrm{~m}$ radius and its center coordinate is $(60,60)$. Based on the scattering forward knowledge, the forward modelling has been established to analyse the response of the coal seam geological model. The forward modelling results are as shown in Fig. 10, where the electrical field distribution is consistent with the model in Fig. 1 and the area with the water-bearing rock is obvious, which means that the bearing water rock is very sensitive to the scattering field.

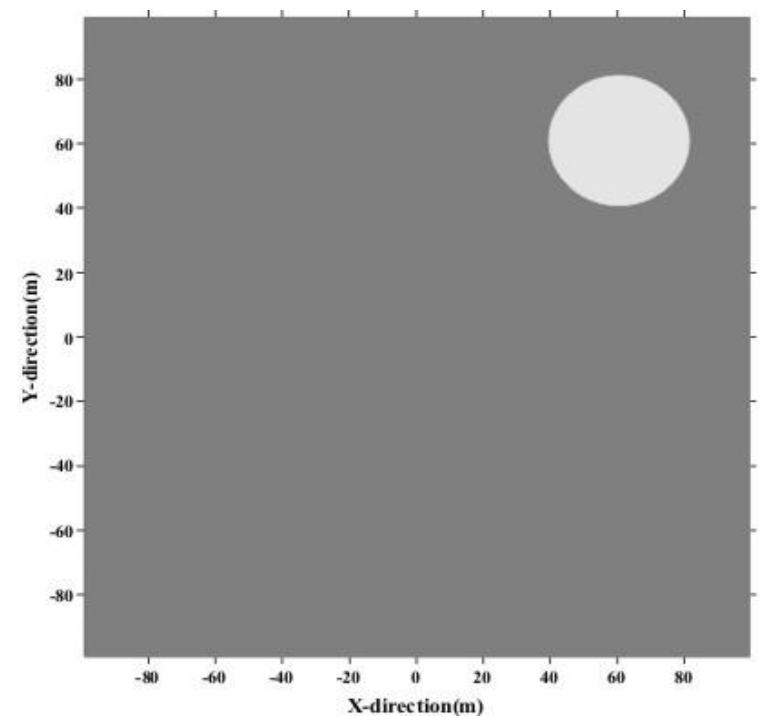

Fig. 9. Model with water-bearing zone of circular shape with the center coordinate being $(60,60)$.

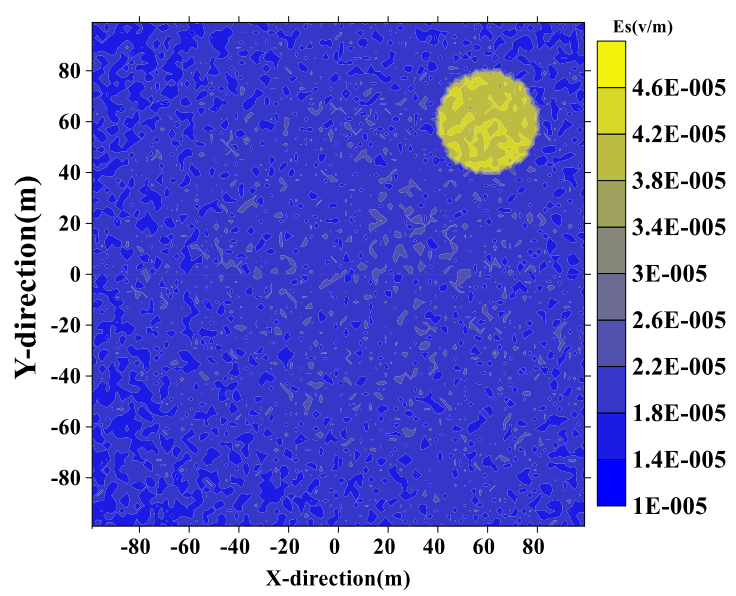

Fig. 10. Distribution of scattering electromagnetic field of Fig. 9.

According to the underground coal mine environment, the observation system is designed to receive information of the scattering field and all the receivers are arranged in the tunnel around the coal seam as shown Fig. 11. It is reasonable to receive scattering field to inverse the permittivity distribution of the coal seam. The inversion modelling result is shown in Fig. 12, where the distribution 
of the permittivity can be used for analysing potential area of the water-bearing rock distribution.

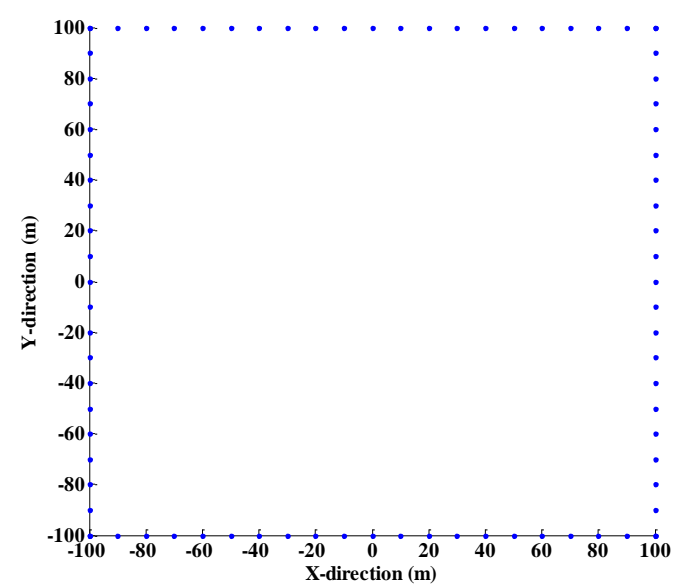

Fig. 11. Observation system in the underground coal tunnel.

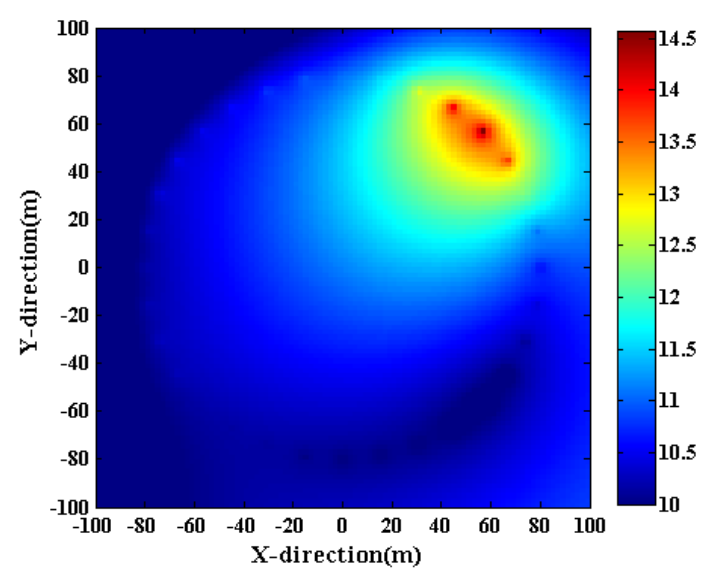

Fig. 12. Inversion modelling result of geological model as shown in Fig. 9.

The similar researches have been done in China State Key Lab of Coal Resources and Safe Mining. The observation points of working face are shown in Fig. 13. The points were set with intervals of $10 \mathrm{~m}$ at the working face or coal laneway. Figure 14 shows the observation results, the electromagnetic radiation intensity indicating earth stress distribution [22]. The curve (Fig. 14) can be used to estimate possible horizontal position of disaster source by electromagnetic radiation intensity. However, our research inversion results can locate the exact position of disaster source in the inside of coal seam as shown in Fig. 12.
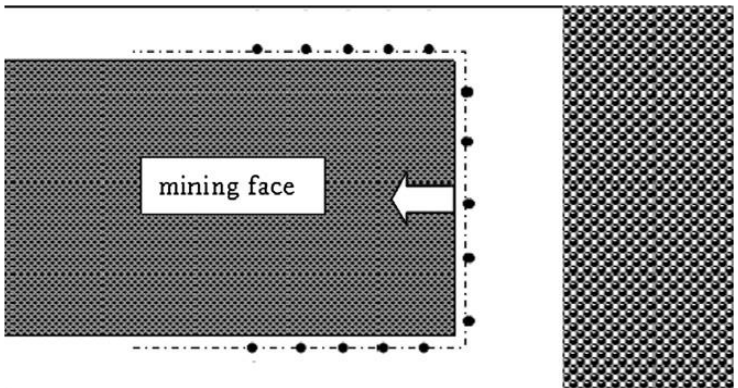

Fig. 13. Schematic of the layout of electromagnetic radiation (EMR) observation points in the working face [20].

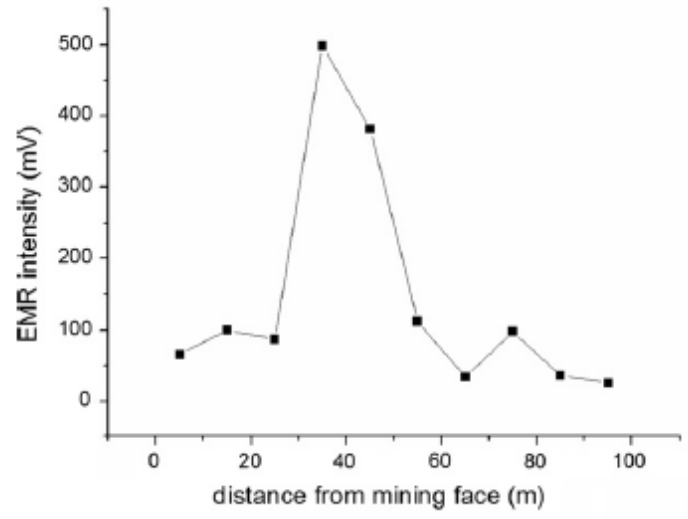

Fig. 14. Distance from mining face [20].

\section{CONCLUSIONS}

This paper uses the distribution of the permittivity to detect the water-bearing rock distribution. Numerical simulations have been carried out to evaluate the proposed method. The main conclusions of the analysis results can be drawn as follows.

1. The results of forward modelling show that the scattering electromagnetic field responses of the anomalous geological bodies are obvious. Specifically, the sensitivity of the scattering electromagnetic field responses is very useful for water-bearing rock detection. The forward modelling shows that it is possible to use the scattering electromagnetic field to investigate the waterbearing rock in the coal seam. Besides, the inversion results match well with the theoretical model.

2. Although the radiation scattering in the electromagnetic field has been studied by many researchers, all of them focus on small scale objects, such as meters, centimetres or millimetres. The target of this research focuses on the coal mine with scale of hundreds of meters. Therefore, the results of the research proved feasibility of using scattering electromagnetic field to solve the geological problem and broadened application range.

3. Comparison between results from China State Key Lab of Coal Resources and Safe Mining and our research results shows that our research can much more precisely locate the position of disaster source in the coal seam.

\section{ACKNOWLEDGMENT}

We are grateful to the anonymous reviewers of this paper.

\section{CONFLICTS OF INTEREST}

The authors declare that they have no conflicts of interest.

\section{REFERENCES}

[1] N. Bojarski, "Electromagnetic inverse scattering", in Proc. of 1971 Antennas and Propagation Society International Symposium, 1971. DOI: 10.1109/APS.1971.1150992.

[2] F. Cakoni and E. Darrigrand, "The linear sampling method for the inverse electromagnetic scattering problem for screens", in Proc. of Applied Mathematics and Mechanics (Pamm) , 2010, vol. 5, no. 1, pp. 645-646. DOI: 10.1002/pamm.200510299.

[3] A. Merfeldas, D. Andriukaitis, D. Gailius, A. Valinevicius, V. 
Markevicius, D. Navikas, M. Zilys, "Field Uniformity and TEM Mode Verification in GTEM 1000 Cell", in Proc. of ELEKTRO 2020, Taormina, Italy, 2020, pp. 1-4, DOI 10.1109/ELEKTRO49696.2020.9130123.

[4] O. M. Bucci and T. Isernia, "Electromagnetic inverse scattering: Retrievable information and measurement strategies", Radio Science, vol. 32, no. 6, pp. 2123-2137, 1997. DOI: 10.1029/97rs01826.

[5] A. Merfeldas, P. Kuzas, D. Gailius, Z. Nakutis, M. Knyva, A. Valinevicius, D. Andriukaitis, M. Zilys, and D. Navikas, "An Improved Near-field Magnetic Probe Radiation Profile Boundaries Assessment for Optimal Radiated Susceptibility Pre-Mapping", Symmetry, vol. 12, no. 7, p. 1063, Jun. 2020. DOI: 10.3390/sym 12071063 .

[6] D. Jaggard and P. Frangos, "The electromagnetic inverse scattering problem for layered dispersionless dielectrics", IEEE Transactions on Antennas \& Propagation, vol. 35, no. 8, pp. 934-946, 1987. DOI: 10.1109/TAP.1987.1144206.

[7] D. L. Colton, "A simple method for solving electromagnetic inverse scattering problems", in Mathematical Methods in Scattering Theory and Biomedical Technology. Addison Wesley Longman Limited, 1998, pp. 3-11. DOI: 10.1088/0266-5611/14/3/012.

[8] M. S. Zhdanov, Geophysical Inverse Theory and Regularization Problems. Elsevier, 2002.

[9] R. Zoughi, Microwave Non-Destructive Testing and Evaluation Principles. Springer, 2012.

[10] G. H. Zalzal, S. R. Shott, R. Towbin, and R. T. Cotton, "Value of CT in the diagnosis of temporal bone diseases in children", Laryngoscope, vol. 96, no. 1, pp. 27-32, 1986. DOI: 10.1288/00005537-198601000-00005.

[11] W. Cai, G.-y. Si, and Q. Zhang "Application of electromagnetic radiation in coal mine and its prospects", Shaanxi Coal, 2009. DOI: 10.3969/j.issn.1671-749X.2009.05.042.

[12] L. M. Dou, J.-C. Tian et al., "Research on electromagnetic radiation rules of composed coal-rock burst failure", Chinese Journal of Rock Mechanics \& Engineering, vol. 24, no. 19, pp. 3541-3544, 2005.
DOI: 10.3321/j.issn:1000-6915.2005.19.023

[13] V. Frid, "Rockburst hazard forecast by electromagnetic radiation excited by rock fracture", Rock Mechanics \& Rock Engineering, vol. 30, no. 4, pp. 229-236, 1997. DOI: 10.1007/BF01045719.

[14] D. Song, E. Wang, Ch. Wang, and F. Xu, "Electromagnetic radiation early warning criterion of rock burst based on statistical theory", Mining Science and Technology (China), vol. 20, no. 5, pp. 686-690, 2010. DOI: 10.1016/S1674-5264(09)60263-5.

[15] M. Li, S. Liu, B. Su, Y. Ma, and Q. Sun, "Study on transient electromagnetic response of high resistivity goafs and its application", Elektronika ir Elektrotechnika, vol. 25, no. 1, pp. 3135, 2019. DOI: 10.5755/j01.eie.25.1.22733.

[16] Y. Ma, J. Shen, B. Su, Y. Ma, and Q. Sun, "Research on ground of penetrating radar in the coal mine detecting: A case study of application in Huaibei coal mine", Elektronika ir Elektrotechnika, vol. 25 , no. 5, pp. 37-42, 2019. DOI: 10.5755/j01.eie.25.5.24354.

[17] T. Lu, Sh.-d. Liu, B. Wang, R.-x. Wu, and X.-w. Hu, "A review of geophysical exploration technology for mine water disaster in China: Applications and trends", Mine Water \& the Environment, vol. 36, no. 5, pp. 331-340, 2017. DOI: 10.1007/s10230-017-0467-z.

[18] M. S. Zhdanov, Geophysical Electromagnetic Theory and Methods. Elsevier, 2009

[19] Z. Liu and S. Liu, Mine Geophysical Exploration. China University of Mining and Technology, 2016.

[20] Y. Zhong, M. Lambert, D. Lesselier, and X. Chen, "A new integral equation method to solve highly nonlinear inverse scattering problems", IEEE Transactions on Antennas \& Propagation, vol. 64, no 5, pp. 1788-1799, 2016. DOI: 10.1109/TAP.2016.2535492.

[21] X. Chen, Computational Methods for Electromagnetic Inverse Scattering, 1st ed. JohnWiley \& Sons, Singapore Pte Ltd., 2018, ch. 6. DOI: $10.1002 / 9781119311997$

[22] E. Wang, X. He, X. Liu, and W. Xu, "Comprehensive monitoring technique based on electromagnetic radiation and its applications to mine pressure", Safety Science, vol. 50, no. 4, pp. 885-893, 2012. DOI: $10.1016 /$ j.ssci.2011.08.013.

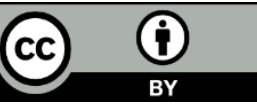

This article is an open access article distributed under the terms and conditions of the Creative Commons Attribution 4.0 (CC BY 4.0) license (http://creativecommons.org/licenses/by/4.0/). 